\title{
Same-Sign Charginos and Majorana Neutralinos at the LHC
}

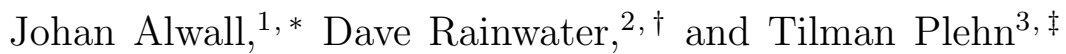 \\ ${ }^{1}$ Theoretical Physics, Stanford Linear Accelerator Center, Menlo Park, CA, USA \\ ${ }^{2}$ Dept. of Physics and Astronomy, University of Rochester, Upstate, NY, USA \\ ${ }^{3}$ SUPA, School of Physics, University of Edinburgh, Scotland
}

(Dated: September 20, 2021)

\begin{abstract}
We demonstrate the possibility of studying weakly interacting new particles in weak boson fusion, using the example of supersymmetric same-sign charginos. This signal could establish the existence of Majorana neutralinos and give access to their electroweak couplings. It can be observed over (supersymmetric) QCD backgrounds provided the charginos are light and not too close to the squark mass. We finally show how same-sign fermion production can be distinguished from samesign scalars or vectors arising in other models of new physics.
\end{abstract}

*Electronic address: alwall@slac.stanford.edu

$\dagger$ Electronic address: rain@pas.rochester.edu

${ }^{\ddagger}$ Electronic address: tilman.plehn@cern.ch 


\section{INTRODUCTION}

In our search for new phenomena, the LHC is about to start a new era of testing the incredibly successful and resilient Standard Model. We know that the Standard Model can only be an effective theory, likely breaking down around the $\mathrm{TeV}$ scale. One of the possible extensions which should appear at that scale is supersymmetry (SUSY) [1]. Its existence would double the particle spectrum, adding a partner of opposite spin statistics for every Standard Model particle.

If supersymmetry exists, it must be broken, as we do not see spin partners of any Standard Model particles [2]. All superpartners must therefore be massive compared to their Standard Model counterparts. Experiments such as LEP and Tevatron [3] have put stringent bounds on many of the SUSY partner masses. The LHC will perform a conclusive search covering masses all the way to the $\mathrm{TeV}$ scale. In the existing literature we find thorough coverage of how to conclusively discover SUSY-like signatures at hadron colliders, primarily via its large production cross sections for the strongly-interacting squarks and gluino [4, 5].

However, discovery is only the beginning of LHC physics - many alternative scenarios of $\mathrm{TeV}$-scale physics can mimic supersymmetry. For a long time we have known how to confirm the Majorana nature of gluinos, provided they are fermions [6]; a similar strategy for Majorana neutralinos is still missing. Serious effort has recently been put into studying how to distinguish between classes of models, mostly by measuring the masses [7] and spins [8] of new particles, mainly in the colored sector. Such spectral data can be used to perform TeV-scale model fits, for example if the spins support a SUSY hypothesis [9]. In comparison, little work has addressed other quantum number measurements at the LHC.

Typical SUSY spectra show gluino and squarks more massive than the non-colored superpartners, due to different QCD v. electroweak gauge coupling evolution from a unification scale, or directly due to the size of the beta functions of the gauge couplings. Such heavy superpartners cascade decay through through successively lighter superpartners, from colored to colorless, until the cascade terminates at the lightest supersymmetric particle, or LSP, which is the dark matter candidate. A typical (long) squark decay radiates first a quark to shed its color charge, and then two leptons to finally arrive at the LSP. If the gluino is heavier than the squarks, it will have the same decay chain, plus an extra quark.

While we can accurately measure masses from the decay kinematics in long cascades, we do not gain any information about the coupling strengths of the intermediate states, save that they're large enough to keep the superpartners from being long-lived - but this is not a truly useful constraint. For top quarks, the corresponding issue is resolved via singletop production, and the analogous process for stops and sbottoms can establish that the stop-sbottom- $W$ boson interaction is an electroweak gauge vertex [10].

Our goal is to observe non-colored superpartner production directly at LHC, to test those superpartners' electroweak character and study their quantum numbers independently of cascade decays. While Drell-Yan production at the LHC is generally lost in the SM and SUSY backgrounds, a previous work identified weak-boson-fusion production as a potentially viable signal [11, 12]. This channel has been extremely successful in finding ways to study light Higgs bosons, including for example the size [13] and structure [14] of their couplings. In the case of superpartners, the probably most pressing question is the Dirac or Majorana nature of the neutralino sector, which the weak-boson-fusion process will allow us to study. 


\section{SAME-SIGN CHARGINOS}

The production of same-sign charged particles at a hadron collider is in general a remarkable signal. It requires a balancing of charge in the final state - the initial state may have at most charge \pm 1 — which limits it to very few sources. We will explore each source for same-sign charginos in turn, starting with the electroweak production mechanism found in weak boson fusion [11].

In all TeV-scale supersymmetric (MSSM) scenarios, charginos subsequently decay. This may be treated as a separate $1 \rightarrow 2$ on-shell process, which can be included as a branching ratio or as a fully spin-correlated decay chain (and similarly for any further decays of the chargino's daughters). For simplicity, we discuss the various processes and their associated Feynman diagrams only up to the produced charginos; their subsequent decay does not alter any of the production mechanisms or topologies.

\section{A. Weak boson fusion processes}

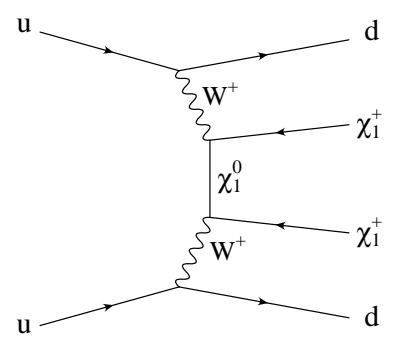

FIG. 1: Feynman diagram for the pure WBF SUSY process $q q^{\prime} \rightarrow q q^{\prime} \tilde{\chi}_{1}^{+} \tilde{\chi}_{1}^{+}$as described in the text. The complete set of diagrams sums over all Majorana neutralinos in the $t$-channel.

In pure weak boson fusion (WBF) a pair of incoming quarks each emit a weak gauge boson: $W^{ \pm} W^{ \pm}$for our case of interest. Because of the massive gauge boson propagators, the scattered quarks acquire a transverse momentum typically of the scale of the $W$ mass, $p_{T}>m_{W}$. This is large enough to make the scattered quarks visible as jets in the detector, albeit at typically small scattering angles, thus far forward and backward in the detector. Particles produced in the fusion process are typically central in the detector, at nearly right angles to the beam axis, and with similarly high transverse momentum.

Because charginos are fermions, their same-sign production via gauge boson fusion must be mediated by a $t$-channel neutral Majorana fermion, to provide the necessary fermion number violation. In the MSSM there are four neutralinos. For each quark-flavor subprocess there are 8 Feynman diagrams of the topology shown in Fig. 1. This set of diagrams is separately gauge invariant.

WBF same-sign chargino production is most significant for a wino pair [11], since charged higgsinos have a much smaller coupling to $W$ bosons. In most MSSM scenarios in agreement with the LEP2 limits, the mixing from the SUSY eigenstates to the mass eigenstates is fairly small. In that sense the observation of the WBF signature could establish the gauginohiggsino nature of the $\tilde{\chi}_{1}^{ \pm}-\tilde{\chi}_{2}^{ \pm}$hierarchy, an important piece of information in reconstructing the supersymmetric Lagrangian. 


\section{B. Non-WBF electroweak processes}
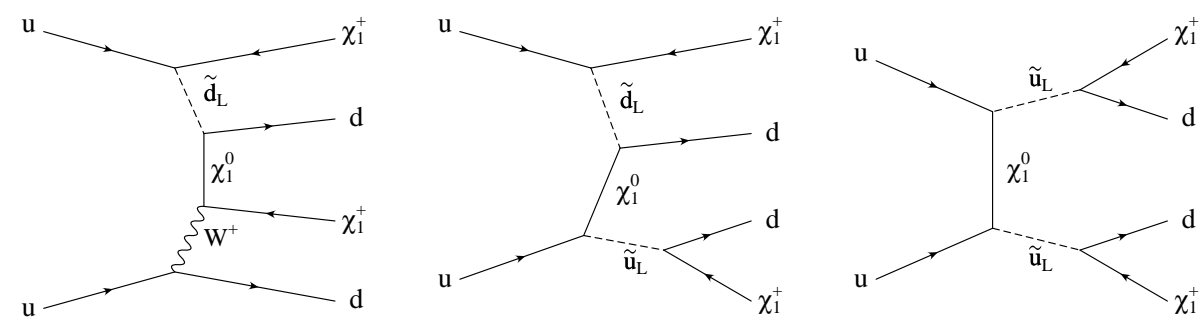

FIG. 2: Representative Feynman diagrams for the electroweak non-WBF SUSY process $q q^{\prime} \rightarrow$ $q q^{\prime} \tilde{\chi}_{1}^{+} \tilde{\chi}_{1}^{+}$. The complete set sums over all Majorana neutralinos in the $t$-channel.

The same final state as for WBF processes can occur via electroweak processes involving non-WBF diagrams, shown by the representative Feynman diagrams of Fig. 2, We observe non-resonant $t$-channel diagrams, singly-resonant squark and doubly-resonant squark processes. The latter numerically dominate, but to properly account for off-shell effects while maintaining gauge invariance we perform a complete calculation. This completeness will become important once we impose kinematic cuts to suppress on-shell squarks.

\section{QCD processes}
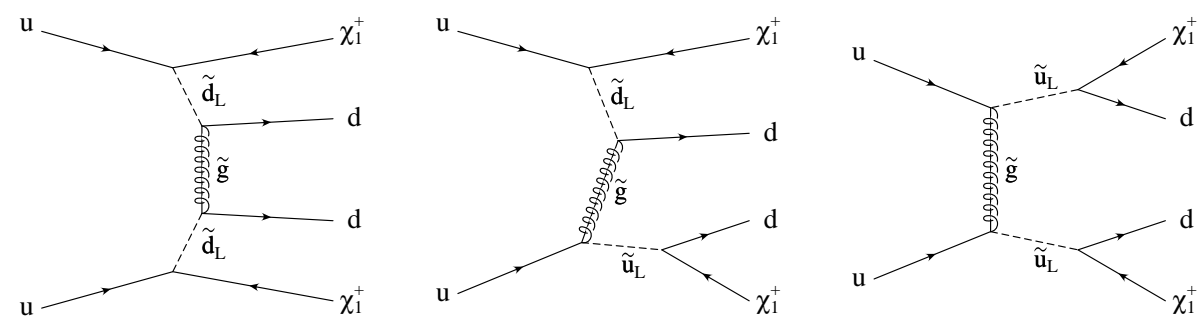

FIG. 3: Representative Feynman diagrams for the QCD SUSY process $q q^{\prime} \rightarrow q q^{\prime} \tilde{\chi}_{1}^{+} \tilde{\chi}_{1}^{+}$.

The dominant background before any kinematic cuts arises from cascade decays of heavy colored squarks (and gluinos, if heavier than squarks), as discussed in the introduction; see Fig. 3, For example, LHC will provide an enormous flux of pairs of valence $u$ quarks, which can scatter to a pair of same-sign up squarks via a $t$-channel Majorana gluino. ${ }^{1}$ Gluino pairs may also decay to same-sign squarks, giving the same final state modulo extra jets; likewise for squark-gluino mixed production. The different processes might be distinguished using the jet multiplicity [15]. All QCD processes occur at huge rates compared to both electroweak sources of like-sign charginos, despite the higher final-state masses and consequent phase space suppression. As in the electroweak non-WBF case, the doubly-resonant component dominates, but we include all possible QCD amplitudes to correctly account for off-shell effects.

\footnotetext{
${ }^{1}$ Same-sign charginos (or same-sign leptons, if charginos decay promptly) from QCD processes can be taken as evidence of the Majorana nature of the gluino [6], once its fermionic spin character is established [8].
} 


\begin{tabular}{|c||l|l|l|l|l|l|l|l|l|l|}
\hline SPS & $1 \mathrm{a}$ & $1 \mathrm{~b}$ & 2 & 3 & 4 & 5 & 6 & 7 & 8 & 9 \\
\hline$\chi_{1}^{+} \chi_{1}^{+}$ & 0.93 & 0.22 & 0.48 & 0.23 & 0.51 & 0.57 & 0.067 & 0.077 & 0.31 & 0.88 \\
\hline$\chi_{1}^{-} \chi_{1}^{-}$ & 0.28 & 0.056 & 0.13 & 0.058 & 0.14 & 0.16 & 0.017 & 0.020 & 0.083 & 0.25 \\
\hline
\end{tabular}

TABLE I: Cross sections [fb] for WBF opposite-sign and same-sign chargino pair production at LHC, for all MSSM benchmark SPS points, without cuts, from Ref. [11]. Cross sections are shown to two significant digits.

\section{SIGNAL AND BACKGROUNDS}

We begin by reviewing the WBF same-sign chargino cross sections calculated in Ref. [11]; the results are repeated in Table I for convenience. With the exception of a few SPS points [16], the cross sections are comparable, of order $1 \mathrm{fb}$ and falling mostly in a range of a factor of three of each other. These leading-order total cross sections are without cuts. Observation at LHC would depend on the rate for a given final state, which would typically require leptons for charge identification. If the chargino decays to lepton plus slepton, this could be done with high efficiency (near 100\% if electron or muon, less for tau). If instead it decays to $W$ boson plus neutralino (typically the lightest one, the LSP), there would be a larger hit in signal rate due to the requirement to observe leptonic $W$ decays.

In some MSSM scenarios, notably anomaly-mediated supersymmetry breaking, the chargino is long-lived due to a near-degeneracy with the lightest neutralino, the LSP. Longlived charged massive particles, or CHAMPs, are searched for at the Tevatron and would be readily observable [17] in the LHC detectors, ATLAS [18] and CMS [19]. Heavy gluinos from gluon fusion are produced very close to threshold, so one has to require $\beta \gtrsim 0.6-0.8$. In the case of WBF charginos this captures the bulk of the signal for charginos light enough to be produced at a sufficient rate, so we do not explicitly impose such a cut.

Our stable-chargino scenario provides the "best" possible signal. First, because of the very high efficiency to capture such events in data. Second, because cascade decay chains are fully reconstructible, so all superpartner masses would be known. We can impose tailored invariant-mass cuts to remove the squark and gluino backgrounds. We examine this scenario first as a baseline to all others, but will find that backgrounds are generally not a problem even when the chargino cannot be reconstructed. In general, we will not focus on the kinematics of the centrally produced charginos — instead, we follow the spirit of a similar Higgs-coupling analysis [14] and rely on the tagging-jet kinematics to analyze the events.

Specifically, we investigate the benchmark point SPS9 [16], an anomaly-mediated supersymmetry-breaking scenario with naturally long-lived wino-like charginos $\tilde{\chi}_{1}^{ \pm}$. The lighter chargino has a mass of $197.4 \mathrm{GeV}$ and the light-flavor squark masses are $1.3 \mathrm{TeV}$, with a lighter gluino. Gluino production is not a background, because gluinos decay to either a top quark plus stop or bottom quark plus sbottom with a radically different final state which can be easily vetoed. We emphasize that SPS9 is only a toy example to demonstrate the utility of this signature with a minimum of complication. Our analysis is equally valid in any other scenario, provided the production rate for same-sign charginos (or their decay products after branching ratios) is sufficient for observation at high luminosity.

For all our calculations we use the event generator MADEVEnT4 [20] with its MSSM extension [11]. We consistently utilize the leading-order parton densities CTEQ6L1 [21]. For all electroweak processes we select the minimum transverse momentum of the tagging 


\begin{tabular}{|l|c|c|c|}
\hline cuts & WBF cuts & $\left|m_{j \chi}-m_{\widetilde{q}}\right|>30 \mathrm{GeV}$ & $\left|m_{j \chi}-m_{\widetilde{q}}\right|>50 \mathrm{GeV}$ \\
\hline \hline All EW & $1.138(0.286) \mathrm{fb}$ & $0.847(0.226) \mathrm{fb}$ & $0.786(0.213) \mathrm{fb}$ \\
\hline WBF & $0.825(0.220) \mathrm{fb}$ & $0.766(0.206) \mathrm{fb}$ & $0.724(0.197) \mathrm{fb}$ \\
\hline EW non-WBF & $0.261(0.053) \mathrm{fb}$ & $41.4(8.52) \mathrm{ab}$ & $23.1(4.76) \mathrm{ab}$ \\
\hline QCD & $0.259(0.040) \mathrm{fb}$ & $8.70(1.58) \mathrm{ab}$ & $3.66(0.775) \mathrm{ab}$ \\
\hline$S / B$ & $1.6 / 1(2.4 / 1)$ & $15 / 1(20 / 1)$ & $27 / 1(36 / 1)$ \\
\hline
\end{tabular}

TABLE II: LHC cross sections for the WBF same-sign chargino signal $\tilde{\chi}_{1}^{+} \tilde{\chi}_{1}^{+} j j\left(\tilde{\chi}_{1}^{-} \tilde{\chi}_{1}^{-} j j\right)$, electroweak and QCD backgrounds at SPS9, for various levels of kinematic cuts described in the text. We also show the signal-to-background ratio $S / B$.

jets as the factorization scale, $\mu_{F}=\min \left(p_{T}(j)\right)$. For QCD processes we use the squark mass for the factorization and renormalization scales, $\mu_{F}=\mu_{R}=m_{\widetilde{q}}$, as suggested by NLO calculations [5].

In addition to an assumed $b$ jet veto to remove (supersymmetric) heavy-flavor backgrounds, we apply the usual weak-boson-fusion cuts for the tagging jets. On top of those we require minimal cuts for the charginos to satisfy detector requirements for observability and tracking. We expect these chargino cuts to have a similar effect as cuts on possible chargino decay products. None of our later results depend in any way on the chargino cuts. The basic level cuts consist of minimum transverse momentum and maximum absolute rapidity:

$$
p_{T}(j)>20 \mathrm{GeV}, \quad|\eta(j)|<4.5, \quad p_{T}\left(\tilde{\chi}_{1}^{ \pm}\right)>10 \mathrm{GeV}, \quad\left|\eta\left(\tilde{\chi}_{1}^{ \pm}\right)\right|<2.5 .
$$

To make use of the inherent characteristics of WBF particle production, namely forwardscattered quark jets with large rapidity separation between them, and central production of the electroweak objects, we impose a jet separation cut and require the colorless objects to lie between the jets [22]:

$$
\left|\eta\left(j_{1}\right)-\eta\left(j_{2}\right)\right|>3.0, \quad \eta(j)_{\min }<\eta_{\tilde{\chi}_{1}^{ \pm}}<\eta(j)_{\max } .
$$

Additionally, we impose an invariant mass cut on all combinations of one jet with one chargino around the known squark mass, which may easily be done for long-lived massive charged particles [17]. We study two more-or-less aggressive versions of this cut, which almost completely removes the QCD and electroweak squark-production backgrounds:

$$
\left|M\left(j, \tilde{\chi}_{1}^{ \pm}\right)-m_{\widetilde{u}}\right|>30(50) \mathrm{GeV} .
$$

This cut of course assumes long-lived charged particles. We give results for both options, as well as only WBF cuts, to show how cut optimization may affect signal rate and the signal-to-background ratio, $S / B$.

\section{A. Cross sections}

Our cross section results for $\tilde{\chi}_{1}^{+} \tilde{\chi}_{1}^{+} j j$ and $\tilde{\chi}_{1}^{-} \tilde{\chi}_{1}^{-} j j$ production at LHC with various levels of kinematic cuts for SPS9 are shown in Table [I]. The QCD and electroweak non-WBF backgrounds are each slightly less than half the size of the signal already after basic WBF 
cuts. The excellent ratio $S / B$ is promising, even when the number of signal events is small. In a long-lived chargino scenario the event may be completely reconstructed, allowing the imposition of an invariant mass cut to remove the squark poles. If such a rejection cut is possible, the backgrounds become truly negligible.

If the charginos decay, this cut would likely turn into something like a transverse-mass cut with lower efficiency. Moreover, there would be some efficiency loss in selecting leptonic final states, but standard techniques in WBF [22] would provide for further significant reduction of the backgrounds. Such generalization is however beyond the scope of this first paper.

Given that the efficiency to tag two forward jets as well as the two central charged tracks is collectively about $60 \%$ [22], our signal requires the full LHC luminosity of $300 \mathrm{fb}^{-1}$, especially to obtain good statistics in the kinematic distributions. WBF production of exotic particles is a natural case for the high-luminosity environment of the SLHC [23], when parameter studies will become more and more the focus for the experiments. However, forward-jet tagging at those luminosities is not yet fully understood, so we limit ourselves to the LHC design luminosity before its planned upgrade. There, we expect a few hundred signal events with high purity - more than enough to perform "precision" measurements in kinematic distributions, given negligible backgrounds. The rate uncertainties would be around $\mathcal{O}(5 \%)$ statistically, probably with similar systematic uncertainties. Parton-density and higherorder QCD uncertainties are known to be of that size or smaller from WBF Higgs and vector boson studies [24].

\section{B. Kinematic distributions}

We show two useful kinematic distributions of the final-state forward tagging jets in Fig. 4. as well as the total deposited transverse energy of all observed objects, $H_{T}=\sum_{i} E_{T_{i}}$, and the jet-chargino invariant mass. For these plots, we impose only the WBF cuts of Eqs. (112). All curves are normalized to unity to emphasize the gross distinguishing characteristics of WBF v. non-WBF electroweak and QCD production. As noted earlier, the backgrounds arise primarily from heavy squark decays, so they give much harder jets (and charginos) for a typical mass separation. This is also the reason for the backgrounds' far larger $H_{T}$ compared to the WBF continuum signal. The leading chargino transverse momentum distribution is almost identical to that for the leading tagging jet. Other standard WBF distributions, such as the azimuthal angle between the tagging jets, $\triangle \phi_{j j}$, are only marginally discriminating.

In a long-lived chargino scenario, all these distributions may be used to suppress the backgrounds to a truly negligible level relative to the signal, as we found in Table II. If the chargino instead decayed promptly, then all curves in $H_{T}$ would shift to the left by some likely universal amount, to account for the unobserved LSP pair. The point is that while all of these distributions would change, the shifts would be very similar for signal and backgrounds, thus retaining the same basic distinctions and separating power. Only the invariant mass of a chargino plus the leading jet is valid exclusively for long-lived scenarios. But, as can be seen from the other distributions, obtaining even better $S / B$ ratios achieved with the first level of standard WBF cuts would be straightforward, also with decays.

We thus do not anticipate any serious background issues, at least in scenarios where the squarks are appreciably heavier than the charginos. For many SUSY models, $S / B$ will be of order 1/1 from the WBF cuts alone, Eqs. (12). Very little effort would be needed to enhance the signal v. background separation further. 

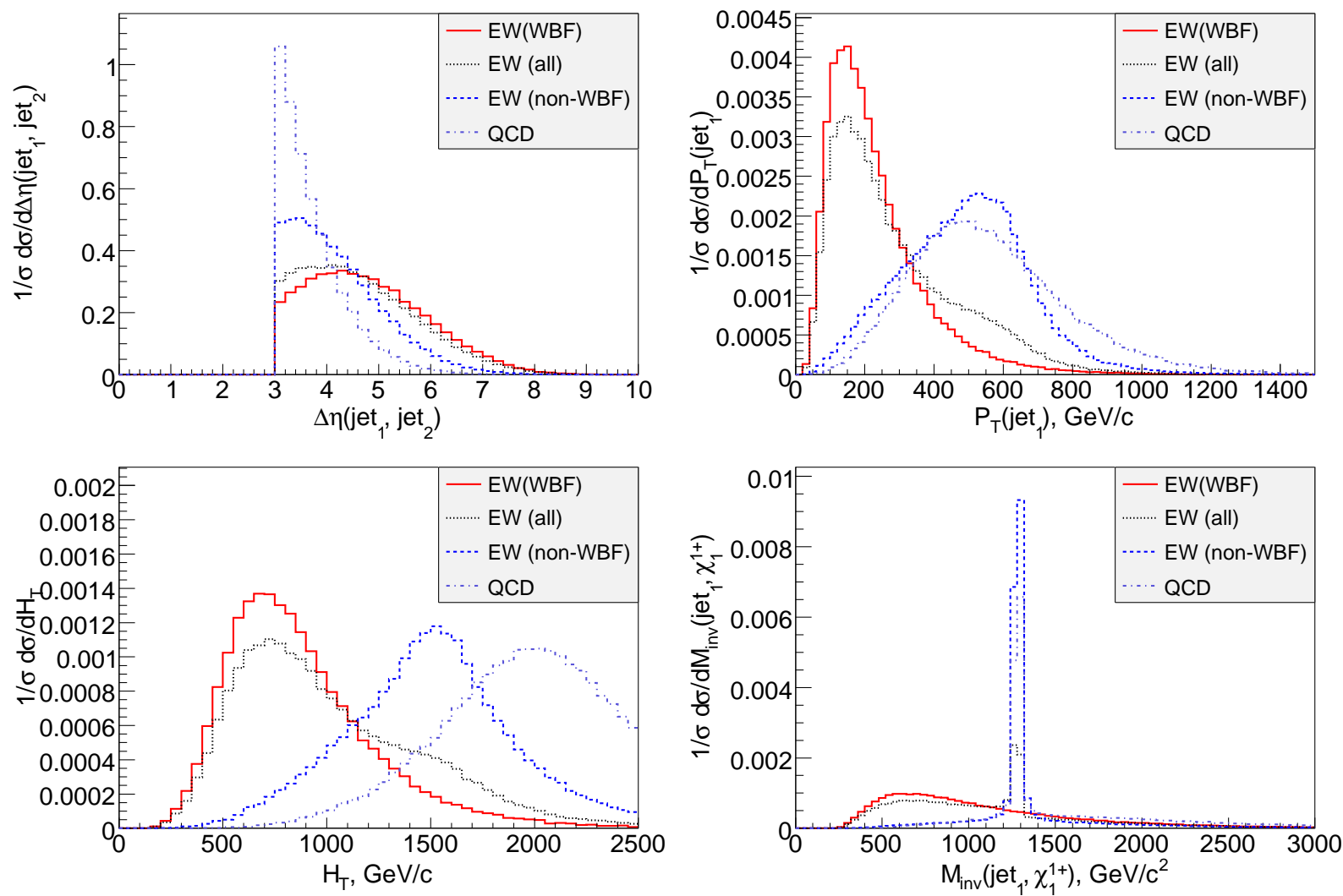

FIG. 4: Distributions for the WBF same-sign chargino signal at SPS9, including the electroweak and QCD backgrounds from squark production. The jets are ordered according to their transverse momentum. Only WBF cuts Eqs. (12) are used. The invariant mass distribution can be used for long-lived charginos only. All distributions are normalized to unity; see Table I for total rates.

\section{DISCRIMINATING BETWEEN NEW-PHYSICS MODELS}

Making new physics discoveries at the LHC immediately means facing the arduous task of determining what we actually see. If we observe same-sign charged particles in weak boson fusion we cannot simply assume that they are supersymmetric charginos; alternative hypotheses must be tested to reach a meaningful conclusion. We already know that other objects can at first glance appear to be gluinos or squarks [25]. Even if heavy colored particle decays were determined to be of the right spin for supersymmetry [8], it can be hard to determine chargino and neutralino candidate spins in cascade decays, not to mention the Majorana or Dirac nature of such weakly interacting new fermions. Our goal is to show that kinematic distributions can be used to discriminate between fermionic same-sign particle production in WBF, and scalars or vectors. (We ignore higher-spin states in good taste.)

To formulate our stable-scalar hypothesis, we use the MSSM two-Higgs doublet model, as implemented in MADEVEnT4 [11, 20]. A general two-Higgs doublet model (also implemented) could be used as well, but this does not change the spin structure, and in any case we consider only normalized distributions. For the spin hypothesis comparison we assume the charged Higgs to be stable on detector timescales due to a near mass degeneracy with its decay products; it may also decay promptly, and all kinematic distributions alter in a way similar to that described for the fermionic chargino case in Sec. IIIB. 
For the vector case, we implement a generic model with a neutral $Z^{\prime}$ and a charged $W^{\prime}$ pair. If done rigorously, this is not entirely straightforward, as we would need to begin with a larger gauge group, such as $S U(2)_{L} \times S U(2)_{R}$, and break it to $S U(2)_{L}$. We would also have to be careful about any additional matter content, which may be necessary depending on the underlying group structure and the breaking mechanism [26]. Since we normalize the scalar and vector cross sections to the fermionic chargino rate and analyze exclusively normalized distributions, we do not worry about such details. Instead, we use a toy model based on an additional Little-Higgs-type gauge sector $W^{\prime} / Z^{\prime}$ with $T$ parity, which makes the $W^{\prime}$ stable on detector timescales. To preserve unitarity at high energies, we include a $T$-odd scalar $H^{\prime}$ and $T$-odd heavy quarks $u^{\prime}, d^{\prime}$, etc. The Feynman rules are the same as for the corresponding Standard Model vertices in all cases. With our $H^{\prime}$ we verify unitarity conservation in the process $W^{+} W^{+} \rightarrow W^{\prime+} W^{\prime+}$. At the LHC, we find that for unitary WBF $W^{\prime+} W^{\prime+} j j$ production the $T$-odd Higgs is not necessary; removing it does not yield a noticeable change in results, because the parton densities restrict the cross section at energies where the Higgs exchange becomes important. However, fermionic partners must be present for our coupling structure. They provide the gauge cancellations necessary for unitarity at energies well below a strong dynamics scale, as will be discussed below. Note that not all Little Higgs models contain all these states; instead, strong dynamics is expected to appear at the few-TeV scale.

To limit our analysis to actual spin effects, we set all final-state same-sign charged particles masses to the chargino mass of SPS9 $(197 \mathrm{GeV})$. Moreover, we normalize all rates, as is common in similar LHC spin studies [8]. We recognize that in general new-physics scenarios, charged scalars and charged vectors are unlikely to be long-lived. However, as stressed above, our analysis in no way relies on this assumption. To make this obvious we show distinguishing kinematic distributions only for the tagging jets. It turns out that they alone can clearly discriminate between the various spins.

Fig. [5] shows four distributions for the two forward tagging jets: two angular correlations and two transverse momenta. All of them are independent of the long-lived nature of the charged particles. We first notice that the scalar case is markedly different from either the fermion or vector cases in all distributions. This arises from the virtual $W$ boson emitted from the incoming quarks. The scalar sector couples to the longitudinal (Goldstone) mode of the virtual $W$ boson, which has a distinct preference for small-angle emission, i.e. a more forward, low- $p_{T}$ tagging jet. Fermions and vectors have no such preference, so the transverse modes contribute much more prominently. If we consider a quark with energy $E$ radiating a vector boson with energy $x E$ and transverse momentum $p_{T}$, the probability of collinear radiation of a transverse or longitudinal $W$ boson can be approximated by [27]:

$$
\begin{aligned}
& P_{T}\left(x, p_{T}\right) \sim \frac{g_{V}^{2}+g_{A}^{2}}{8 \pi^{2}} \frac{1+(1-x)^{2}}{x} \frac{p_{T}^{2}}{\left(p_{T}^{2}+(1-x) m_{W}^{2}\right)^{2}} \longrightarrow \frac{g_{V}^{2}+g_{A}^{2}}{4 \pi^{2}} \frac{1+(1-x)^{2}}{2 x} \frac{1}{p_{T}^{2}} \\
& P_{L}\left(x, p_{T}\right) \sim \frac{g_{V}^{2}+g_{A}^{2}}{4 \pi^{2}} \frac{(1-x)^{2}}{x} \frac{m_{W}^{2}}{\left(p_{T}^{2}+(1-x) m_{W}^{2}\right)^{2}} \quad \longrightarrow \frac{g_{V}^{2}+g_{A}^{2}}{4 \pi^{2}} \frac{(1-x)^{2}}{x} \frac{m_{W}^{2}}{p_{T}^{4}}
\end{aligned}
$$

The couplings $g_{A, V}$ describe the gauge coupling of the $W$ bosons to the incoming quarks. The last approximation assumes large transverse momentum $p_{T} \gg(1-x) m_{W}$, describing the upper end of the $p_{T}$ spectrum. In this limit the radiation of longitudinal $W$ bosons falls off sharper than the radiation of transverse $W$ bosons, i.e. , the tagging jets associated with Higgs production are softer than the tagging jets associated with fermionic charginos 

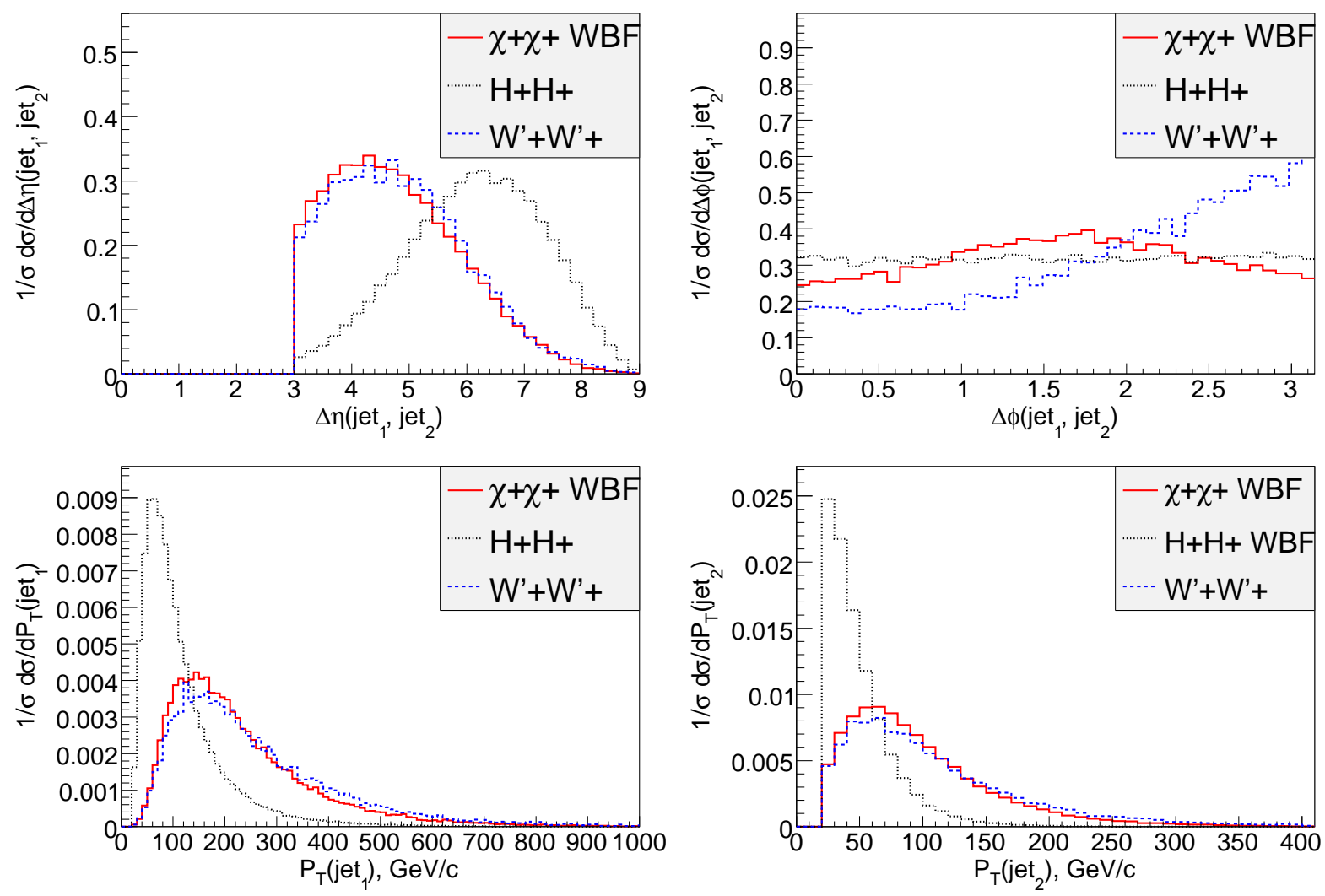

FIG. 5: Kinematic distributions for the tagging jets in WBF production of charginos, scalars and vectors. None of the distributions rely on the charged particles being long-lived.

or with vectors. This is confirmed in the first, third and fourth panels. Note that in the more realistic of the $x$ limits, $x \ll 1$, where the tagging jets carry most of the energy, there is no difference in the $x$ behavior of the two spectra. Unfortunately, none of these three distributions distinguishes between objects which couple to the transverse modes, i.e. between fermions and vectors, which can be understood from this simple approximation.

The second panel of Fig. 5 saves the day. It shows the azimuthal angular separation, which has a slight enhancement for charginos at $\triangle \phi_{j j}=\pi / 2$. In contrast, for the vector case we see a factor of three difference in rate between $\triangle \phi_{j j}=0$ and $\pi$. The flat scalar curve reflects the lack of spin information being passed from one incoming quark current to the other, from a $t$-channel neutral scalar Higgs boson or 4-point $W W H H$ interaction. As seen in Fig. 6, double scalar production behaves exactly like single-scalar production [14]. Thus $\Delta \phi_{j j}$ is the one distribution we find which distinguishes the fermion and vector cases - and both from the scalar case.

Because of the gross differences in all distributions between scalar and higher-spin cases, identifying scalar production would be straightforward, probably requiring less luminosity for good statistical discrimination. Discriminating between fermion and vector cases is also straightforward, provided one does not cut on $\triangle \phi_{j j}$ to reduce backgrounds.

In the general spirit of our analysis we do not use any distributions for the charged objects themselves. Just as in the single-Higgs case [14], the truly useful information is fortunately encoded in the forward tagging jets. 

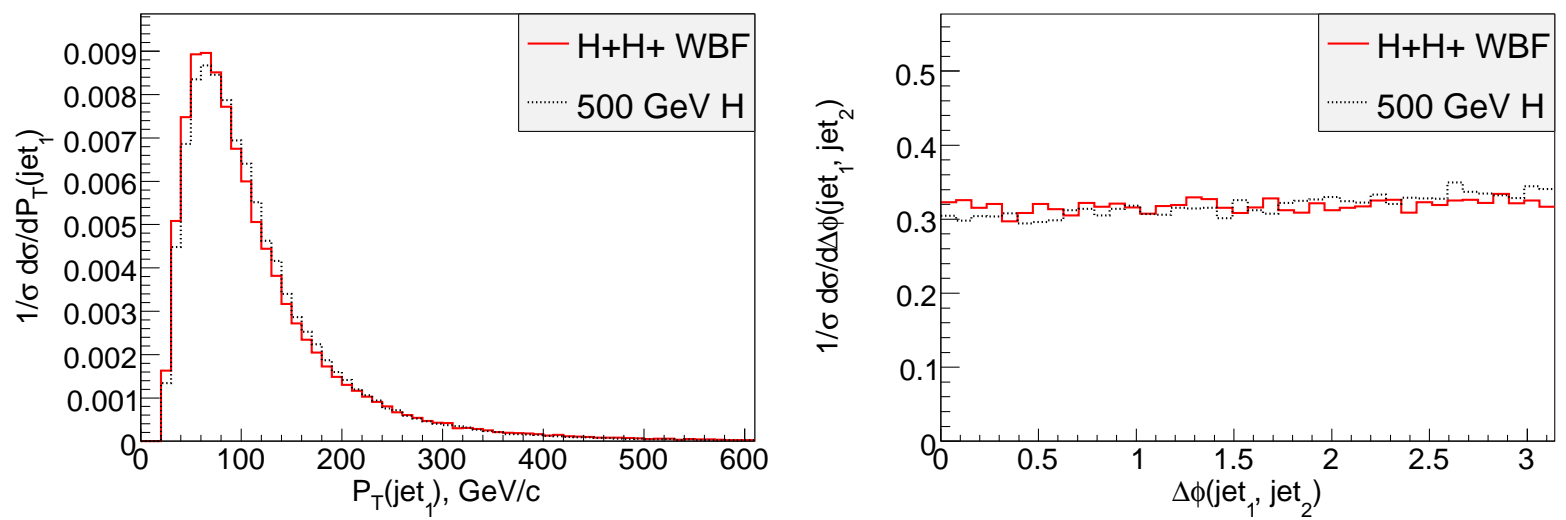

FIG. 6: Distributions comparing charged scalar pair production with a single neutral scalar of $500 \mathrm{GeV}$, to demonstrate identical spin structure.
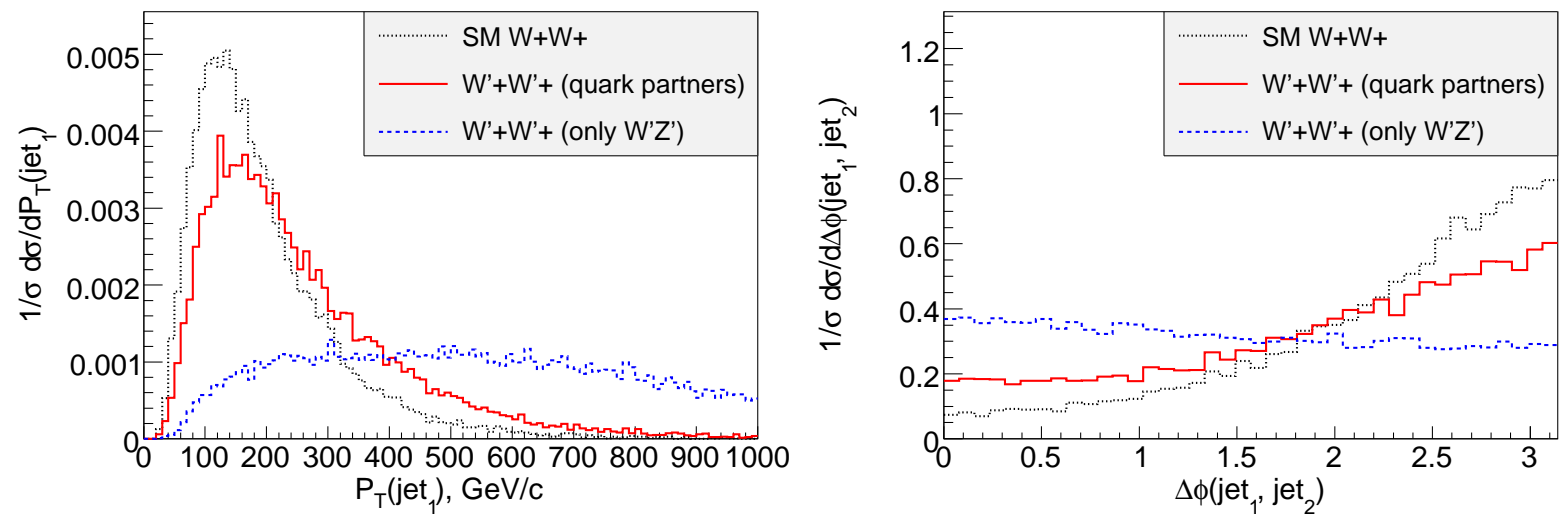

FIG. 7: Comparison between Standard Model $W$ pairs, $W^{\prime}$ pairs, both with the full set of Feynman diagrams and the $W^{\prime}$ case (wrongly) omitting all heavy quark diagrams. Bad high-energy behavior can clearly be seen in the transverse momentum distribution of the left panel. The lack of gauge cancellations also leads to incorrect angular distributions (right).

We should briefly comment on an aspect of models with additional vectors, e.g. Little Higgs or universal extra dimensions. Often these models contain a discrete parity, like $R$ parity in supersymmetry, to provide a dark matter particle. We find that it is crucial to include fermionic partners of the quarks in such cases, as in our toy vector model. If these are left out, gauge cancellations between WBF and $W^{\prime}$ Bremsstrahlung diagrams (as occur in the Standard Model) are spoiled, producing anomalous high transverse momentum and invariant mass distributions for the jets and vectors. The dramatic effect of neglecting heavyquark diagrams on the transverse momentum distributions and on the angular correlation can be seen in Fig. 7. We do not perform a full analysis here, but given the bad highenergy behavior of the jet (and chargino) transverse momentum, it seems possible that signs of unitarity violation may appear well below the scale of assumed strong dynamics in such models. Additionally, a lack of gauge cancellations produces (very) incorrect angular distributions, as seen in the right panel. 


\section{CONCLUSIONS}

In order to expand the capability of the LHC to explore the electroweak sector of new physics scenarios, we have examined electroweak production of supersymmetric same-sign charginos in weak boson fusion. Typical signal cross sections are known to be in the femtobarn range, small but definitely viable for long-term measurements, not intended to supplant discovery. Typically, large differences in mass scales between charginos and squarks provide for excellent suppression of SUSY-electroweak and SUSY-QCD backgrounds to the level of the signal, already with simple weak-boson-fusion acceptance cuts.

Observing this signal would most importantly provide direct confirmation that at least one neutralino is a Majorana fermion. However, that assumes that the charged particles produced are fermions. We therefore showed that LHC can indeed distinguish scalar, fermion and vector same-sign production in weak boson fusion, using only kinematic distributions of the forward tagging jets - most notably the azimuthal angle between them. That the tagging jet encode all the necessary information to discriminate between different spin hypotheses is fortuitous: this renders our analysis ultimately independent of whether the heavy charged particles are quasi-stable or decay promptly.

For our discrete-parity vector toy model, we encountered an interesting aspect, that to maintain gauge invariance we need parity-odd partners of the quarks. This might have implications for Little Higgs models with $T$ parity, many of which do not contain those quark partners. Our calculations suggest that unitarity violation have visible effects well below the strong dynamics scale of these models, where one would assume that new physics controls the behavior.

One caveat for more general scenarios is that there will be a Standard Model background from $W^{+} W^{+} j j$ production, which is $\mathcal{O}(100)$ fb with WBF-style cuts. After leptonic branch-

ing ratios, it would be within a factor of a few of the SUSY cross section. A detailed calculation with decays is beyond the scope of this paper, but we expect many kinematic differences to appear between the signal and Standard Model background, in both lepton momentum and angular distributions.

\section{Acknowledgments}

This research was supported in part by the Swedish Research Council (JA), the U.S. Department of Energy under grant No. DE-FG02-91ER40685 (DR). We thank Tom Rizzo, Tim Tait and in particular Kaoru Hagiwara for useful discussions on many aspects discussed in this paper, and Joe Lykken for providing an incentive to speed up. T.P. would like to thank the DESY theory group for their hospitality where this paper was finalized. D.R. would like to thank the SUPA ultra-mini workshop series for their support during his stay in Edinburgh. 
[1] For reviews of supersymmetry, see: S. P. Martin, arXiv:hep-ph/9709356;

I. J. R. Aitchison, arXiv:hep-ph/0505105.

[2] E. L. Berger, B. W. Harris, D. E. Kaplan, Z. Sullivan, T. M. P. Tait and C. E. M. Wagner, Phys. Rev. Lett. 86, 4231 (2001);

U. Nierste and T. Plehn, Phys. Lett. B 493, 104 (2000);

P. Janot, Phys. Lett. B 564, 183 (2003).

[3] See e.g. :

http://www-cdf.fnal.gov/physics/exotic/exotic.html for CDF and http://www-d0.fnal.gov/public/new/new_public.html for D $\varnothing$.

[4] S. Dawson, E. Eichten and C. Quigg, Phys. Rev. D 31, 1581 (1985).

[5] W. Beenakker, R. Höpker, M. Spira and P. M. Zerwas, Nucl. Phys. B 492, 51 (1997);

W. Beenakker, M. Krämer, T. Plehn, M. Spira and P. M. Zerwas, Nucl. Phys. B 515, 3 (1998);

W. Beenakker et al., Phys. Rev. Lett. 83, 3780 (1999).

[6] R. M. Barnett, J. F. Gunion and H. E. Haber, in Proc. of the Summer Study on High-Energy Physics in the 1990s, Snowmass 1988, ed. S. Jensen; H. Baer, X. Tata and J. Woodside, ibid.;

H. Baer, X. Tata and J. Woodside, Phys. Rev. D 41, 906 (1990);

R. M. Barnett, J. F. Gunion and H. E. Haber, Phys. Lett. B 315, 349 (1993);

V. D. Barger, W. Y. Keung and R. J. N. Phillips, Phys. Rev. Lett. 55, 166 (1985).

[7] H. Bachacou, I. Hinchliffe and F. E. Paige, Phys. Rev. D 62, 015009 (2000);

B. C. Allanach, C. G. Lester, M. A. Parker and B. R. Webber, JHEP 0009, 004 (2000);

B. K. Gjelsten, D. J. Miller and P. Osland, JHEP 0412, 003 (2004);

B. K. Gjelsten, D. J. Miller and P. Osland, JHEP 0506, 015 (2005);

D. J. Miller, P. Osland and A. R. Raklev, arXiv:hep-ph/0510356.

[8] A. J. Barr, Phys. Lett. B 596, 205 (2004);

A. J. Barr, arXiv:hep-ph/0511115;

J. M. Smillie and B. R. Webber, JHEP 0510, 069 (2005);

M. Battaglia, A. K. Datta, A. De Roeck, K. Kong and K. T. Matchev, arXiv:hep-ph/0507284;

P. Meade and M. Reece, Phys. Rev. D 74, 015010 (2006);

C. Athanasiou, C. G. Lester, J. M. Smillie and B. R. Webber, JHEP 0608, 055 (2006) and arXiv:hep-ph/0606212,

J. M. Smillie, arXiv:hep-ph/0609296,

A. Alves, O. Eboli and T. Plehn, arXiv:hep-ph/0605067;

L. T. Wang and I. Yavin, arXiv:hep-ph/0605296;

A. Alves and O. Eboli, arXiv:hep-ph/0704.0254;

S. Y. Choi, K. Hagiwara, Y. G. Kim, K. Mawatari and P. M. Zerwas, Phys. Lett. B 648, 207 (2007) arXiv:hep-ph/0612237.

[9] R. Lafaye, T. Plehn and D. Zerwas, arXiv:hep-ph/0404282;

P. Bechtle, K. Desch and P. Wienemann, arXiv:hep-ph/0412012.

[10] D. Berdine and D. Rainwater, Phys. Rev. D 72, 075003 (2005).

[11] G. C. Cho, K. Hagiwara, J. Kanzaki, T. Plehn, D. Rainwater and T. Stelzer, Phys. Rev. D 73, 054002 (2006).

[12] A. Datta, P. Konar and B. Mukhopadhyaya, Phys. Rev. Lett. 88, 181802 (2002).

[13] M. Dührssen et al., Phys. Rev. D 70, 113009 (2004).

[14] T. Plehn, D. L. Rainwater and D. Zeppenfeld, Phys. Rev. Lett. 88, 051801 (2002);

V. Hankele, G. Klamke, D. Zeppenfeld and T. Figy, Phys. Rev. D 74, 095001 (2006).

[15] A. Freitas, P. Skands, M. Spira and P. M. Zerwas, arXiv:hep-ph/0703160. 
[16] B. C. Allanach et al., in Proc. of the APS/DPF/DPB Summer Study on the Future of Particle Physics (Snowmass 2001) ed. N. Graf, Eur. Phys. J. C 25, 113 (2002).

[17] B. C. Allanach, C. M. Harris, M. A. Parker, P. Richardson and B. R. Webber, JHEP 0108, 051 (2001);

W. Kilian, T. Plehn, P. Richardson and E. Schmidt, Eur. Phys. J. C 39, 229 (2005);

for a detailed analysis see:

M. Fairbairn, A. C. Kraan, D. A. Milstead, T. Sjostrand, P. Skands and T. Sloan, Phys. Rept. 438, 1 (2007).

[18] ATLAS TDR, report CERN/LHCC/1999-15 (1999).

[19] CMS TDR, report CERN/LHCC/2006-001 (2006).

[20] F. Maltoni and T. Stelzer, JHEP 0302, 027 (2003);

J. Alwall et al., arXiv:0706.2334 [hep-ph], to appear in JHEP.

[21] J. Pumplin, D. R. Stump, J. Huston, H. L. Lai, P. Nadolsky and W. K. Tung, JHEP 0207, $012(2002)$.

[22] S. Asai et al., Eur. Phys. J. C 32S2, s19 (2004); and references therein.

[23] F. Gianotti et al., Eur. Phys. J. C 39, 293 (2005).

[24] See e.g. : T. Han, G. Valencia and S. Willenbrock, Phys. Rev. Lett. 69, 3274 (1992);

T. Figy, C. Oleari and D. Zeppenfeld, Phys. Rev. D 68, 073005 (2003);

G. Bozzi, B. Jäger, C. Oleari and D. Zeppenfeld, Phys. Rev. D 75, 073004 (2007).

[25] H. C. Cheng, K. T. Matchev and M. Schmaltz, Phys. Rev. D 66, 056006 (2002);

A. Datta, K. Kong and K. T. Matchev, Phys. Rev. D 72, 096006 (2005) [Erratum-ibid. D 72, 119901 (2005)];

A. Datta, G. L. Kane and M. Toharia, arXiv:hep-ph/0510204.

[26] For a review see e.g. :

M. Schmaltz and D. Tucker-Smith, Ann. Rev. Nucl. Part. Sci. 55, 229 (2005).

[27] T. Han, arXiv:hep-ph/0508097. 